\title{
Size Enlargement of Pharmaceutical Powders by Wet Agglomeration and Drying Techniques $\dagger$
}

\author{
H. Leuenberger \\ Pharmazeutisches Institut der Universität, Basel *
}

\begin{abstract}
Today, tablets are still the most desired form of dosage due to the convenient administration, the chemical and physical stability of the drug in a solid state, and the high production rate of a tabletting machine based on the compaction of powders or granules. Because direct compression vehicles are still in the minority, pharmaceutical powders have to be processed in order to obtain a tailored particle size distribution exhibiting a good flowability, compressibility, compactibility, wettability, lack of dust, etc. Thus, the size enlargement by wet agglomeration and drying of pharmaceutical powders is an important unit operation in a batch-wise production of granules. For an optimal quality assurance, the starting materials (drug substance, excipients) and the process technologies applied need to be characterized and defined in an appropriate way. Special attention has to be paid to scale-up processes and to monitoring the amount of granulating liquid added to the powder system. Although a batch-wise production is the method of choice from the point of view of a quality assurance department, as a batch can be accepted or rejected, the trend towards lean and just-in-time production focuses the attention on the feasability of continous processes such as a wet agglomeration and drying technique. Besides the subsequent production of tablets, spherical pharmaceutical granules called pellets with a mean particle size in the range of $0.4 \mathrm{~mm}$ to $1.4 \mathrm{~mm}$ are usually filled into hard gelatine capsules. The controlled production of pellets with an appropriate mean size and narrow size distribution is highly desired. Due to environmental protection and safety, the use of distilled or demineralized water as a granulation liquid for the wet agglomeration process is the first choice. However, the design of innovative novel dosage forms, i.e. the modification of powders, may make the use of an appropriate organic solvent may be a prerequisite. Thus, the production of novel dosage forms, of specially tailored properties of granules, may need a special process technology with a solvent recovery system, i.e. a correlation can be established between novel product properties and the use of novel process technologies, such as the vacuumfluidized bed granulation and drying method.
\end{abstract}

\section{Introduction}

In the pharmaceutical industry, powders play an important role. In the majority of cases, the raw materials used, i.e. active substances and excipients, are powders. In addition, approx. $80 \%$ of the medicinal products sold on the world market are solid dosage forms, i.e. tablets, capsules, drag, etc. Thus the process technologies to modify pharmaceutical powders in order to obtain a final dosage form are of special importance. Due to the high potency of drugs, special attention has to be paid to GMP (good manufacturing practice), to the optimal design of the dosage forms and to a strict quality

* Totengässlein 3, CH-4051 Basel, Switzerland

$\dagger$ Received June 14th, 1993 control. Thus the process technologies concerning size enlargement of powder particles need to fulfill requirements which are specific to the area of pharmaceutics. Total quality control and lean production are important issues. Thus the powders need to be characterized and specified according to specially developed standard operation procedures, unit operations such as the moist agglomeration process have to be validated and should usually be supervised for, e.g. a batch documentation by an intelligent in-process control.

For many reasons, a drug substance cannot be administered in its bulk state. Thus it is important to design a drug delivery system, i.e. dosage form, which is tailored to the properties of the 
drug substance and to the mode of administration. As a consequence, the drug powder has to be modified and processed together with auxiliary substances in order to optimize a number of properties such as, e.g. handling, stability, compactibility, solubility and controlled release of the active substance. The development of a controlled release system showing a well-defined release profile as a function of time and an optimal bioavailability of the drug substance means in many cases a real challenge for the scientists involved. In the case of a controlled release system, a sufficient drug concentration for therapeutic use has to be maintained in the systemic circulation for an extended period of time. Thus the patient may need to administer the dosage form only once a day instead of three times. As a consequence, patient compliance and the therapeutic effect can often be substantially improved.

Another important issue is the safety of a dosage form. Special emphasis has to be given to it in the case of a controlled release dosage form, as a failure - where the total amount of drug is released immediately - may lead to toxic side effects or adverse reactions. This problem can be reduced if instead of a single large, non-disintegrating matrixtype dosage form with a high load of active substance, subunits such as pellets, i.e. small and highly sperical granules with a smooth surface coated with a polymer for the controlled release are used. These pellets may even show a pH-dependent release of the active substance, e.g. an aliquot of the pellets could have an enteric coating, i.e. no drug release at low $\mathrm{pH}$ values, e.g. during the residence time in the stomach. Thus a hard gelatine capsule could contain different types of pellets providing a specially tailored drug dissolution in the gastrointestinal tract. Due to the much better distribution of subunits such as pellets or granules within the gastrointestinal tract, the intra-and interindividual variations in the bioavailability of a drug can often be reduced.

Concerning the development of a dosage form, there are numerous technological implications which cannot be treated here in an exhaustive way.

\section{Characterization of particulate matter}

It is much easier to determine the chemical behaviour of particulate matter than to characterize the inherent properties of a powder exhibiting functions such as flowability, compressibility, solubility, inflammability, caking, electrostatic charging, etc. In many cases, these properties cannot be attributed to the powder substance alone, but may be a consequence of a pretreatment, an interaction with moisture content of the surrounding atmosphere, etc. These possible side or main effects have to be kept in mind. In order to complicate the situation even more, properties of the powder can be quantified only with a simultaneous indication of the apparatus and principle used for the measurement of the property in question. Thus the lack of an absolute standard often represents a handicap. Typical examples of such properties are the size distribution (by number, by weight, i.e. sieving), specific surface (BET, Blaine), flowability, etc. Often, the residual moisture content of the powder (adsorbed water, residual content of organic solvent used to crystallize the active substance, etc.) plays an important role.

Recently, [1] Mandelbrot introduced the concept of fractal dimension. This concept is a powerful tool to describe surface characteristics of particles and granules (see Fig. 1a - 1c). Another important concept is the theory of percolation which can be applied in a wide range of research areas [2]. Both concepts are interrelated. Thus different types of particulate aggregation lead to different fractal dimensions of these agglomerates. Actually, both concepts play an important role in the research activities concerning the size enlargement area of pharmaceutical powders. It is important to realize in this respect that a powder mixture behaves differently to a powder consisting of a pure substance. This fact is true with respect to wet and dry agglomeration techniques. In a rough description, a binary powder mixture consisting of different materials (e.g. brittle versus plastic; hydrophilic versus hydrophobic; soluble versus insoluble; pharmacologically active substance versus excipient) compressed to tablets may show important variations in their properties as a function of the mixture ratio, i.e. percolation effects may occur which are comparable to the variation of the electric conductivity in a binary powder system consisting of an electrically conductive material and an insulator. Such effects may become even more pronounced after size enlargement by wet agglomeration and drying techniques with a subsequent production of tablets (see Fig. 2a, $2 \mathrm{~b}$ [3]).

\section{Pocess monitoring in the case of granula- tion by agitation}

Power consumption and/or torque measurements of mixers or rotary granulators as a function of the 


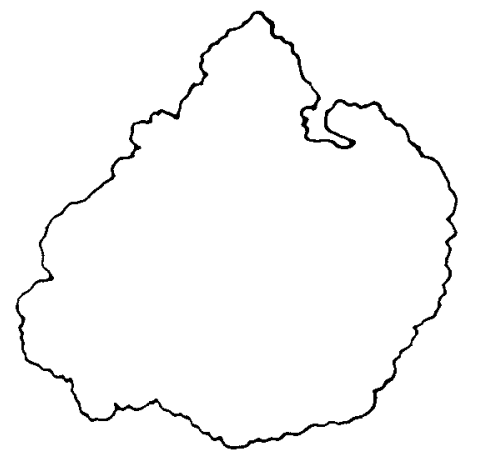

Fig. 1a An outline of the surface roughness of a lactose granule [3]. Coastline of a lactose granule with linear fractal dimension $\mathrm{D}=1.091 \pm 0.007$.

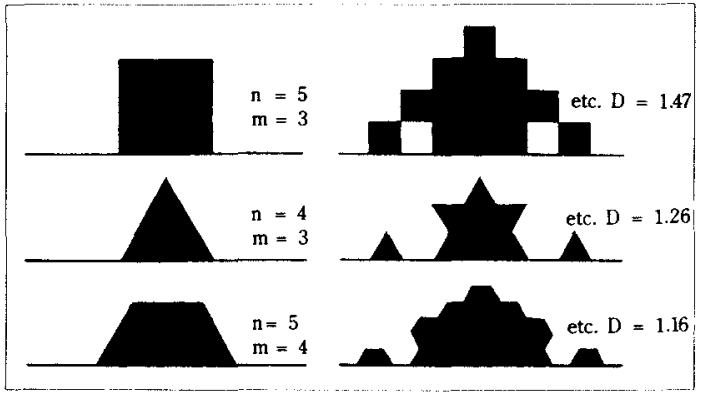

Fig. 1b Mathematical simulation of coastlines with fractal dimensions $\mathrm{D}=1.47,1.26$ and $1.16[3]$.

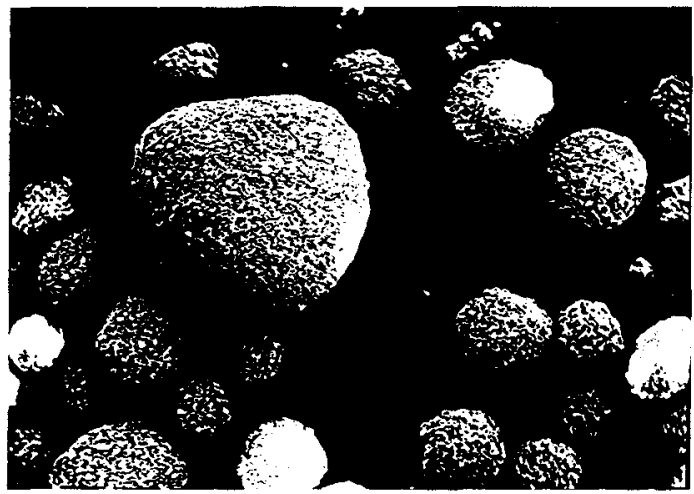

Fig. 1c Scanning electron micrograph of the surface of lactose granules [3].

mass of granulating liquid added per unit time have been used to efficiently control the agglomeration process [4-14]. This type of control facilitates scaleup and scale-down exercises and significantly improves the reproducibility of the desired size distribution of granules and/or pellets. If the amount of granulating liquid cannot be added continuously by a pump but needs to be added at the beginning,
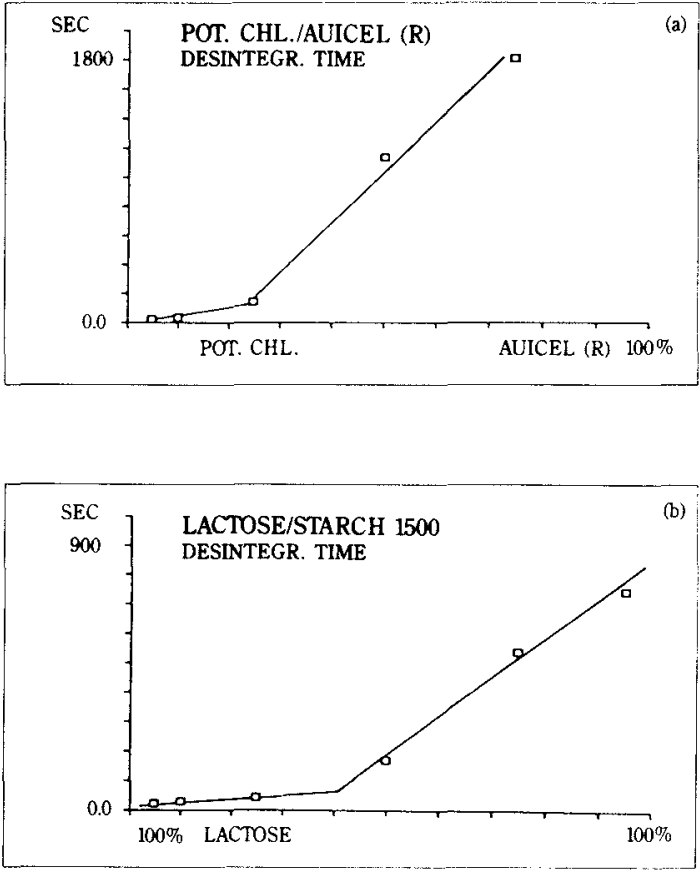

Fig. $2 \mathrm{a}$ and $\mathbf{2 b}$ The disintegration time as a function of compositions of binary component compacts [3].

a) Potassium chloride/Advicel ${ }^{R}$.

b) Lactose/Starch Rx $1500^{\mathrm{R}}$.

i.e. at $t=0$ of the batch production, frequency analysis of power consumption could be the method of choice [10].

Fig. 3 describes a typical power consumption profile. It is important to realize that the mass of granulating liquid has to be metered and added by a pump in a continuous controlled way. Thus a fine tuning of the exact volume of granulating liquid needed is feasible and can be controlled electronically. The amount of granulating liquid to be applied depends on the desired granule size distribution and the intensity of wetting needed, e.g. for a hydrophobic active substance mixed with hydrophilic excipients, etc. It has to be emphasized that the range of mass of granulating liquid which can be added is limited, and corresponds fairly well to the plateau range of the power consumption profile in Fig. 3. Thus it is possible to use the power consumption profile as an analytical tool in the R\&D department: if the granulating liquid is not an appropriate one and, e.g. dissolves too much of the solid material, the pattern of the power consumption will deviate considerably from the normal profile described in Fig. 3. It is also 


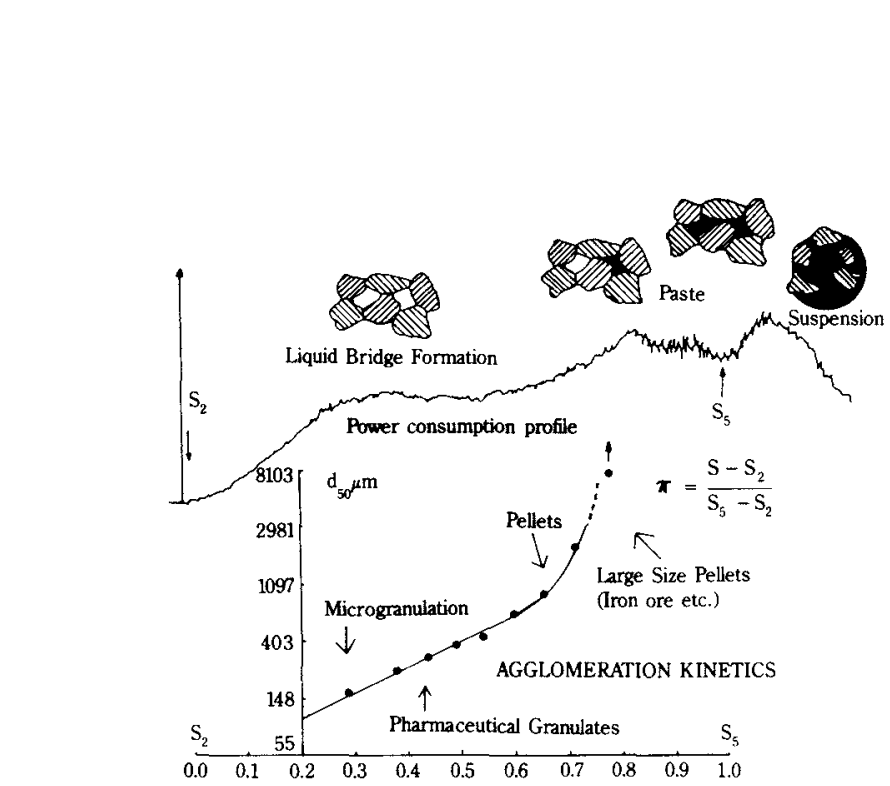

Fig. 3 Typical power consumption profile for the agglomeration of lactose 200 mesh (DMV monohydrate) with $4 \%$ (W/W) polyvinyl-pyrrolidone of binder. The solution is added at a constant rate. $\pi$ Corresponds to the dimensionless amount of granulation liquid added, i.e. to the ratio of interparticulate void space filled up with liquid [12].

possible to detect the formation of, e.g. a hydrate during the granulation process. In such cases, an alternative granulating liquid has to be chosen. Fig. 4 demonstrates the two possible methods to control the granulation process: the absolute power

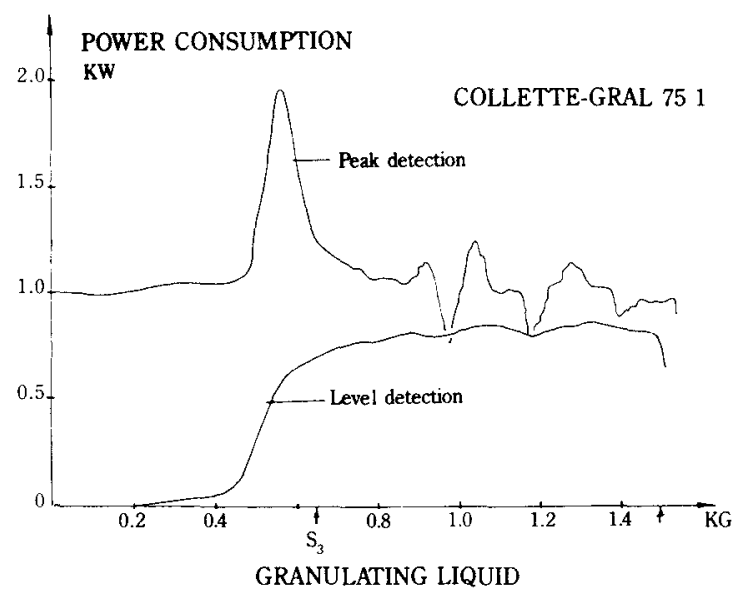

Fig. 4 The amount of granulating liquid added is controlled by the power consumption profile, i.e. by the peak detection mode (differentiated signal) or by the level detection mode [6].

consumption level (1) or the first derivative (2) of power consumption level, i.e. the point of steepest ascent can be used to define the end point of the granulation process. The term "end point of the granulation process" was already used before introduction and implementation of the control of granulation processes by power consumption. It is important to realize that the peak detection signal which is measured by power consumption can still be compared to a "land mark" expressing a defined state of cohesivity of the moistened powder bed. Thus the signal is luckily still ahead of the end point and allows subsequent addition of a predetermined, constant amount of granulating liquid. As the relative position of the control signal of the power consumption profile is directly related to the initial particle size distribution of the primary material and to the initial moisture content, smaller variations of these two parameters are automatically compensated. Thus it is possible to obtain a high reproducibility of the granule size distribution. This type of control has been successfully introduced in the production department at Sandoz Ltd., Basel.

A comparison of granule size distributions $(90 \mu \mathrm{m}-$ $710 \mu \mathrm{m})$ obtained by manually adding a fixed, and by automatically adding an adjusted, amount of granulating liquid shows a better yield for the automatic mode $(91.5 \% \pm 0.4 \%)$ than for the manual mode $(81.0 \% \pm 2.4 \%)$ with a significantly lower standard deviation [14]. It is important to emphasize that the success of this method does not depend on the type of mixer.

\section{A prototype for a quasi-continuous pro- duction of granules}

In the food and chemical industry, continuous production lines play an important role, whereas the pharmaceutical industry is mainly based on a batch-type procedure. Concerning the safety of a dosage form and quality assurance, batches are very convenient. Thus a well-defined batch can be accepted or refused by the quality control department. In the case of a continuous process, a batch has to be defined somewhat artificially, i.e. the amount of product, e.g. the amount of granules produced within 6 to 8 hours. On the other hand, continuous processes offer two important advantages: a) there is no difficult scale-up exercise necessary for larger "batches"; and b) it should be possible to realize a 24 -hour automatic production line.

In order to combine the advantages of batch-type and continuous production, a prototype for a quasicontinuous production line was developed (see Fig. 5). The principle of this quasi-continous production line is based on semi-continuous production of mini-batches in a specially designed high-shear mixer/granulator which is connected to a continuous multicell-fluidized bed dryer.

In order to study the feasibility of such a quasicontinuous production line, a placebo formulation $[4 \%(\mathrm{~W} / \mathrm{W})$ polyvinyl-pyrrolidone (PVP) added as a 
binder in a dry state, $10 \%(\mathrm{~W} / \mathrm{W})$ corn starch and $86 \%(\mathrm{~W} / \mathrm{W})$ lactose 200 mesh] was used. The weighing system which is available on the market was not involved in the first experiments. Thus, a predefined amount of powder of the placebo formulation was added to the twin-type mixer and mixed thoroughly. This amount of powder is then granulated by continuously adding granulating liquid up to a fixed amount. This limit is determined by a moisture content signal which is triggered by the power or torque measurement [6]. The moist granules are then discharged through a screen into the first cell of the multicell-fluidized bed dryer unit to disagglomerate imaginative lumps. Due to the twin-type set-up of the high-shear mixers, the first mixer can be fed with a new mini-batch of powder and be thoroughly mixed in a dry state during the time the powder is being agglomerated by granulating liquid in the second mixer/granulator.

Thus the quasi-continuous production of granules can be described as a train of mini-batches passing the compartments of dry mixing, granulation and drying like parcels. The prototype of the multicell dryer consists of five cells which are designed for different air temperatures, i.e. in the first cell the granules are dried at a high temperature, e.g. $60^{\circ} \mathrm{C}$, and in the last cell, ambient air temperature and humidity can be used to achieve equilibrium conditions.

Due to this principle, a batch defined for quality control purposes consists of a fixed number of $n$ mini-batches. Thus a tight in-process control of the mixing/granulation and drying step provides an excellent "batch" record of the quasi-continuous production of granules, and an excellent opportunity for a continuous validation of the process and the equipment.

\section{The Controlled Production of Pellets}

An alternative to measuring the power consumption consists in measuring the torque of the mixer motor which turns the bottom plate of a rotary granulator [13]. The principle was used in the case of a Glatt fluidized bed rotor granulator (see Fig. 6). Due to the differences in the momentum transfer between the bottom plate and the pellets, the torque profile cannot be compared directly to the power consumption profile in a high-speed mixer. However, it was clearly possible to find a relationship between the median pellet size and the torque value obtained when the continuous addition of granulating liquid
COMBINED WET GPANULATION

AND MULTIPLE CHAMBER DRYING PROCESS

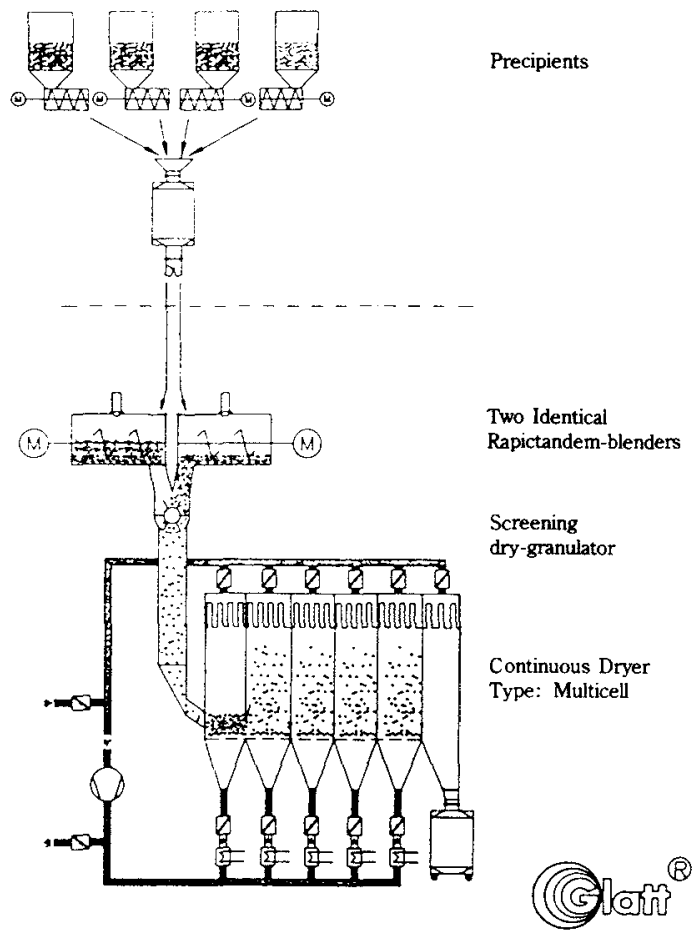

Fig. 5 Flow-chart of a quasi-continous production line for granules

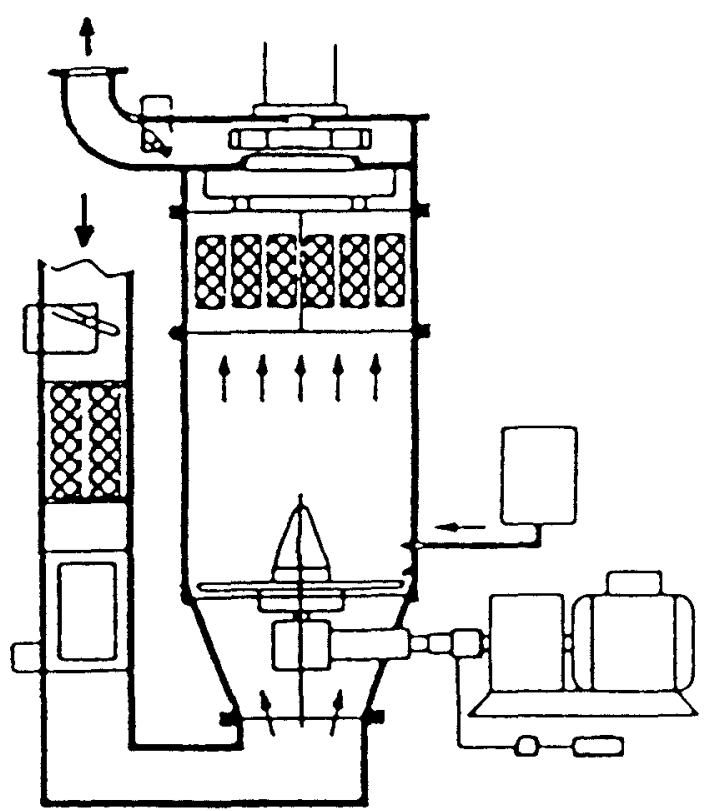

Fig. 6 Glatt Fluidized Bed Rotor Granulator equipped to measure the torque of the bottom plate [13]

was stopped (see Fig. 7). With microcrystalline cellulose and demineralized water, a very narrow pellet size distribution was obtained with the following 


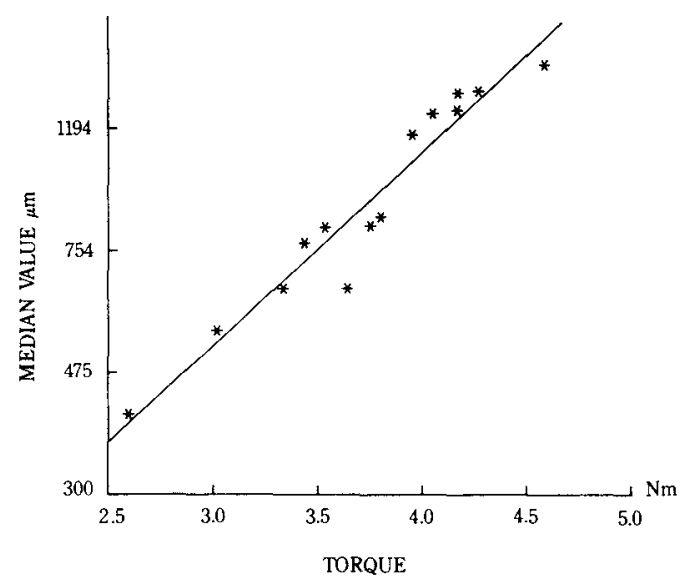

Fig. 7 Median pellet size as a function of the torque measured after stopping addition of granulating liquid.

yield: between $710 \mu \mathrm{m}$ and $1000 \mu \mathrm{m}$ : $72 \%$; between $710 \mu \mathrm{m}$ and $1400 \mu \mathrm{m}: 97 \%$.

It has to be emphasized that these pellets have been produced without adding any seeds. The rationale is the following and follows the same lines as in the case of the controlled production of granules. Due to the possible variations in initial moisture content of the primary material such as microcrystalline cellulose and the variations in moisture content of the inlet air in the rotary-fluidized bed equipment, it is important that the powder material which is used in this process indicates the required amount of granulating liquid needed itself. An alternative way to produce spherical granules is to use a coating procedure to coat seeds (non-pareilles) with active substances and excipients. For this purpose, the same equipment can be used. It is important that the seed particles already have a rather large diameter (e.g. $-300-600 \mu \mathrm{m}$ ) and a narrow size distribution so that finer particles can be layered on the rolling seed particles.

\section{A Novel Fluidized Bed System Operating Under Vacuum Conditions}

Introduction and rationale

This new equipment was designed as a closed-loop system with a solvent recovery facility (see Fig. 8). Thus the advantages of the vacuum drying and of the fluidized-bed process were combined in a unique way. In addition, the superposition of both principles leads to new product properties and consequently to a high potential for innovative and optimized soliddosage forms [15-17, 19].

The use of vacuum

In the case of organic solvents, vacuum drying is a fast process. It is important that the organic solvents are removed to an acceptable extent as regards the

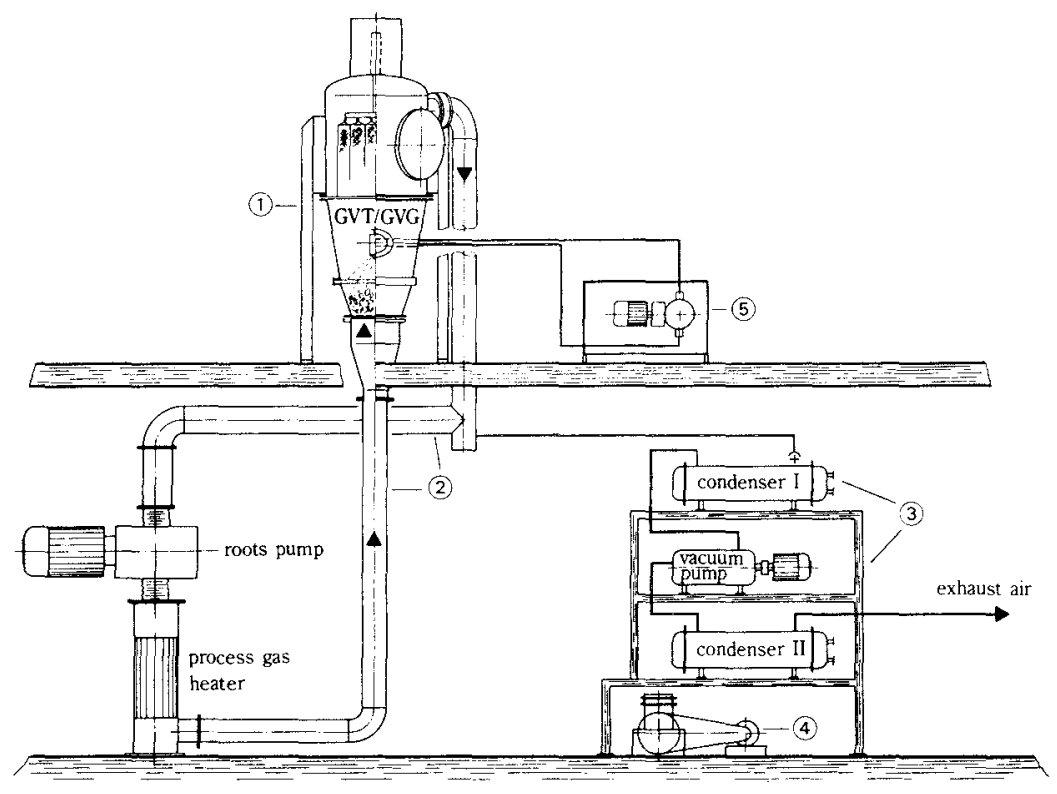

Fig. 8 Vacuum-Fluidized Bed Unit. 3 = solvent recovery system

$1=$ operating tower $\quad 4=$ chiller unit

$2=$ closed-loop system $\quad 5=$ high-pressure spray system 
toxicity of the residual amount.

This process is also advantageous for oxygen and temperature-sensitive materials.

Explosion hazards

Due to lowering the system pressure to below that of the ignition pressure of organic solvents, the use of an inert gas carrier can generally be avoided. However, it has to be taken into account that the ignition pressure of a hybrid system (organic solvent vapour, residual air, powder) may be different from the minimum ignition pressure of the corresponding binary system air/solvent vapour.

Use of the fluidization process

A fluidized bed has the advantage of an excellent heat and mass transfer. Geldart [18] classifies different types of powder material as a function of its fluidization ability, e.g. an extremely wet powder which is very cohesive cannot be fluidized. Thus in such a case, pretreatment by vacuum drying the material on the bottom plate of the product chamber would be the method of choice and emphasizes again the combination of the vacuum drying with a fluidized-bed-drying process.

It is important to realize that a fluidized bed can also be established at rather low pressures such as, e.g. 100 mbar, as the gas velocity can be adjusted to maintain a fluidized bed. In the equation which describes the conditions of the fluidized bed, the gas velocity shows a quadratic dependence (see equation 1), whereas the density $\varrho$ decreases linearly with the system pressure $p$ assuming in a first approximation that the ideal gas equation can be applied (see equation 2).

$$
\begin{aligned}
& \Delta_{\mathrm{p}}=\frac{\mathrm{h}(1-\epsilon) \varrho \mu^{2} \mathrm{gc}}{\mathrm{d}} \\
& \varrho=\frac{\mathrm{RT}}{\mathrm{M}}
\end{aligned}
$$

$\Delta \mathrm{P}=$ differential pressure across fluidized bed

$\mathrm{p}=$ system pressure

$\varrho \quad=$ gas density

$\mathrm{R}=$ gas constant

$\mathrm{T}=$ absolute temperature

$\mathrm{M}=$ molecular weight of the gas

$\mathrm{h}=$ height of fluidized bed

$\epsilon=$ porosity of the fluidized bed

$\mathrm{c}=$ constant

$\mathrm{d}=$ particle diameter

$\mu=$ gas velocity
The Vacuum-Fluidized Bed System

The equipment is described in detail in figure 6 and permits operating the fluidized bed in a closed-loop system. The residual air and/or residual vapour of the organic solvent is used at rather high gas velocities to maintain the fluidized bed of powder. The gas is heated by the process gas heater. The pressure difference $\Delta \mathrm{p}$ to maintain the fluidized bed is achieved with the Roots molecular pump. The solvent vapour is partially removed from the closed-loop system by the solvent recovery unit. This unit consists of two condensers and permits recovery rates of $90 \%$ to $95 \%$ on the low-pressure (vacuum) side, where the recovered solvents show a high purity due to the efficient filter system which prevents particulate contamination. On the high-pressure side, an additional amount of solvent is condensated leading to a total of up to $99 \%$ recovery rates. It is obvious that the additional amount of solvent recovered passed through the oil vacuum pump, and is consequently contaminated by traces of oil, etc. If desired, the exhausted air can be additionally filtered by active carbon, i.e. the equipment meets all known requirements concerning optimal environmental protection. Because the closed-loop system normally operates under vacuum, no inert gas carrier is present which has to be cooled down for solvent recovery purposes and recycled, i.e. heated up again to maintain the classical fluidized bed at atmospheric pressure.

Applications of the Vacuum-Fluidized Bed System

Experiences with drying

In Figs. 9 and 10, the decrease in moisture content is plotted as a function of process time. It is evident that an initially high content of moisture is permitted and that fast drying times can be achieved in the case of organic solvents. "Tailing effects" sometimes represent difficulties (see Fig. 10), where the final drying time can be shortened by purging the system, usually with an inert gas carrier.

The use of inert gas carriers to purge the system

It is of course possible to use an inert gas carrier, e.g. nitrogen, to purge the system. Thus an additional reduction in the residual content of organic solvent in the final product can be achieved. In special cases, the equipment can also be used as an open-loop system in the final drying stage. As the residual content of organic solvent is already low, the requirements of environmental protection can again be easily 
sequently in vivo to a poor bioavailability which is unacceptable. In order to improve the dissolution properties of a poorly soluble drug, drug-solid solutions may be prepared, e.g. by spray drying where the drug is embedded into a hydrophilic matrix which can be easily dissolved in water. Polyvinyl-pyrrolidone (PVP) or polyethylene glycol (PEG) is often used as a hydrophilic matrix and acetone as a solvent if the active substance is soluble in acetone. It is evident that such a preparation needs special attention. For this purpose, the vacuum-fluidized bed equipment was used to optimize the product properties and to study the performance of this system used for a spray agglomeration process. Thus an acetone solution containing PVP and the active substance was sprayed on a powder consisting of microcrystalline cellulose, corn starch and polyplasdone $\mathrm{XL}$. For this study, a $2^{4}$ factorial design was established. In all cases, the active substance was released in water within 5 minutes. The main results of the $2^{4}$ design can be summarized as follows: the mean granule size is increased by a higher system pressure, lower inlet gas temperature, lower spray pressure and higher PVP concentration in the spray solution. Narrow granule size distributions can be achieved with a high system and spray pressure and a low content of PVP in the spray solution. To reduce the residual content of acetone, a low system pressure and a high gas-inlet temperature are advantageous.

met. If desired, an exhaust air-active charcoal filter may be used in addition.

\section{Granulation}

Because no air should be injected into the product chamber, it is evident that a special high-pressure nozzle is needed for the spray solutions (see. Fig. 8). Due to the fact that organic solvents can be used as granulating liquid, a lot of applications in the field of controlled release of active substances and of highly water-sensitive materials become feasible. The following two sections should give a hint concerning the potential for product optimization and for special product properties, which are necessary for product innovation $[19,20]$.

Spray Agglomeration; Spray Drying; Product Optimization

Unfortunately, many biologically very active substances show a low water solubility (e.g. $<0.01 \%$ $\mathrm{W} / \mathrm{V})$. Thus the dissolution of the active drug from a solid-dosage form leads to problems, and sub-

Special Properties Related to the Above VacuumFluidized Bed Process

\section{Granules}

It is important to notice that the granules which were obtained showed a high porosity and a high specific surface. Both the narrow and the broad granule size distribution could be used for tabletting without problems [19]. Due to the aspects of the granules it can be concluded that the spray agglomeration process prevailed. However, if the system pressure is lowered to ca. $100 \mathrm{mbar}$, part of the PVP drug solution is spray-dried leading to typical spherical hollow shells. The samples analysed in an X-ray diffractometer showed that the active substance was present in an amorphous state.

\section{Tablets}

Due to the high porosity of the final tablets, it is not surprising that the active substance was released in water within 5 minutes. The tablets of the sixteen 
$A_{\text {manufactured granule batches of the corresponding }}$ $2^{4}$ design were analysed by mercury porosimetry. The mean pore size of the tablets varied between $1.2 \mu \mathrm{m}$ and $2.4 \mu \mathrm{m}$, and the specific surface between $0.97 \mathrm{~m}^{2} / \mathrm{g}$ and $0.63 \mathrm{~m}^{2} / \mathrm{g}$. Today, it is possible to describe such a system by the concept of fractal geometry. The fractal dimension of the pore system did not change very much and ranged between 2.82 and 2.86. It is interesting to realise that the fractal dimension of a Menger sponge, i.e. of an idealized three-dimensional network of pores (see Fig. 11), is much lower and equal to 2.72. On the other side, the fractal dimension of an idealized bronchial tree (see Fig. 12) is equal to 2.9 .

\section{Coating in Vacuum-Fluidized Bed System}

In order to evaluate the coating performance, metallic magnesium particles (diameter: 90-140 $\mu \mathrm{m})$ were used and coated with Eudragit ${ }^{(1)} \mathrm{S} 100$. Eudragit $^{\circledR} \mathrm{S} 100$ is a methacrylic acid - methyl methacrylate copolymer (1:2) - and is insoluble in $0.1 \mathrm{n}$

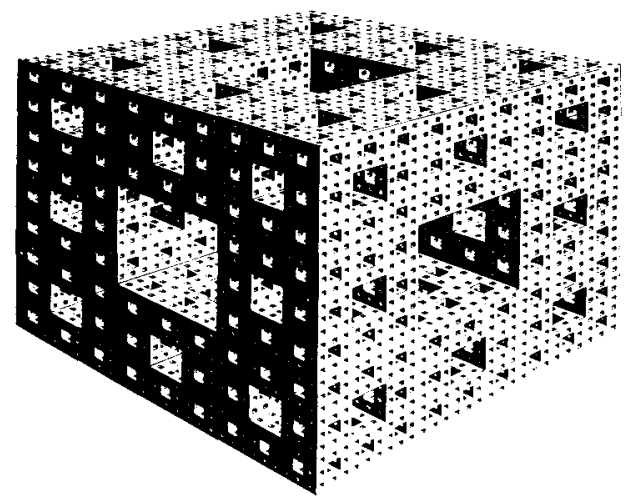

Fig. 11 Menger sponge (idealized 3-dimensional network of pores) with fractal dimension 2.72 [1].

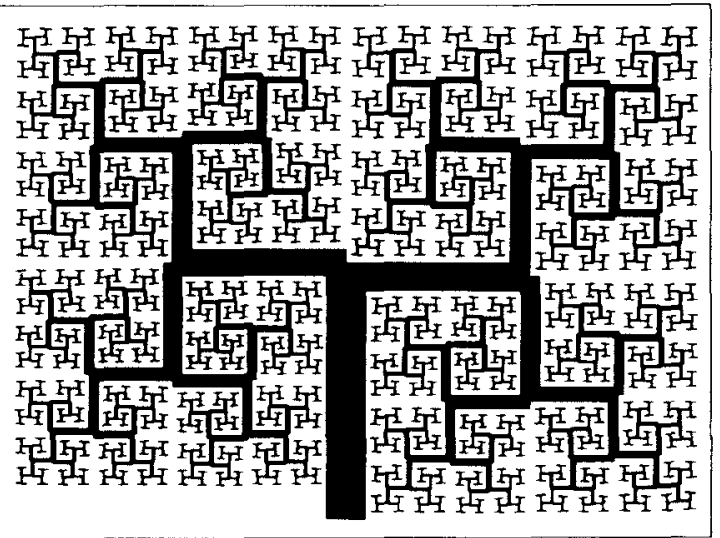

Fig. 12 Representation of an idealized bronchial tree with a fractal dimension of 2.9 [1].
$\mathrm{HCl}$. Thus this film is used for an enteric coating of granules or tablets. To obtain the spray solution, $3.5 \%(\mathrm{~W} / \mathrm{W})$ Eudragit ${ }^{\text {(I) }} \mathrm{S} 100$ was dissolved in an acetone-methanol mixture $(12 \% / 88 \% ; \mathrm{W} / \mathrm{W})$ heated up to a temperature of $90^{\circ} \mathrm{C}$. At a system pressure of ca. 250 mbar and a gas-inlet temperature of $60^{\circ} \mathrm{C}$, the mixture was sprayed from the top onto $9 \mathrm{~kg}$ magnesium powder at a rate of $80 \mathrm{~g} \mathrm{~min}^{-1}$. Thus the thickness of the film on the magnesium particles corresponds to a weight gain of ca. $3 \%$ $(\mathrm{W} / \mathrm{W})$. As metallic magnesium reacts in $0.1 \mathrm{n} \mathrm{HCl}$ extremely fast, i.e. like an explosion of $\mathrm{MgCl}_{2}$ and $\mathrm{H}_{2}$, the amount of $\mathrm{H}_{2}$ gas was measured. An entericcoating quality of these magnesium particles can be obtained for a film thickness corresponding to a minimum of $4 \%(\mathrm{~W} / \mathrm{W})$ of coating material. The coating of the metallic magnesium powder leads to secondary agglomerates with a mean diameter of ca. $310 \mu \mathrm{m}$ exhibiting much better flow properties [17].

\section{References}

1) Mandelbrot, B.: The Fractal Geometry of Nature, W.H. Freeman and Company, New York, 1977.

2) Stauffer, D. and Aharony, A.: Introduction to Percolation Theroy, second edition, Taylor and Francis, 1992.

3) Leuenberger, H.; Holman, L.; Usteri, M. and Winzap, S.: Percolation Theory, Fractal Geometry and Dosage Form Design, Pharm. Acta Helv., 64, (1989), 34-39.

4) Lindberg, N.O.; Leander, L.; Wenngren, L.; Helgesen, H. and Reenstierna, B.: Studies on granulation in a small planetary mixer I, Acta Pharm Suec. 11, (1974), 603-609.

5) Lindberg, N.O.; Leander, L.; Nilsson, P.G. an Reenstierna, B.: Studies on granulation in a small planetary mixer II, Acta Pharm. Suec., 14, (1977), 197-204.

6) Leuenberger, H.: Granulation, New Techniques, Pharm. Acta. Helv. 57, (1982), 72-82.

7) Holm, P.; Schaefer, T. and Kristensen, H. G.: Granulation in High-Speed Mixers, Part V. Power Consumption and Temperature Changes During Granulation, Powder Technology, 43, (1985), 213-223.

8) Terashita, K.; Yasumoto, M. and Miyanami, K.: Determination of End-Point in Agitation Granulation and Powder Coating, Yakugaku Zasshi, 105, (1985), 1166-1172.

9) Terashita, K.; Katou, M.; Ohike, A. and Miyanami, K.: Analysis of End-Point with Power Consumption 
in a high-speed mixer, Pharm. Bull., 38, (1990), 1977-1982.

10) Terashita, K.; Watano, S. and Miyanami, K.: Determination of End-Point by Frequency Analysis of Power Consumption in Agitation Granulation, Chem. Pharm. Bull. 38, (1990), 3120-3123.

11) Leuenberger, H.: Scale-up granulation processes with reference to process monitoring, Acta Pharm. Tech. 29, (4),(1983), 274-280.

12) Leuenberger, H.; Usteri, M.; Imanidis, G.; Winzap, S.: Monitoring the granulation process: granulate growth, fractal dimensionality and percolation threshold, Boll. Chim. Farm. 128, (1989), 54-61.

13) Leuenberger, H.; Luy, B. and Studer, J.: New development in the control of moist agglomeration and pelletization process, S.T.P. Pharma, 6, (5), (1990), 303-309.

14) Dürrenberger, M.; Werani, J.: The Control of Granulation Process by Power Consumption Measurement in Pharmaceutical Production, Proceed. 4th Int. Symp. on Agglomeration Toronto, June 2-5, 1985, C.E. Capes Editor, Iron and Steel Society, Inc. p. 489-496.

15) Luy, B.; Hirschfeld, P. and Leuenberger, H.:
Granulieren und Trocknen in der VakuumWirbelschicht, Pharm. Ind. 51, 1, (1989), 89-94.

16) Luy, B.; Hirschfeld, P. and Leuenberger, H.: Granulation and Drying in Vacuum Fluid Bed Systems, Drugs made in Germany, 32, 3 (1989), 3-8.

17) Leuenberger, H.; Luy, B. and Hirschfeld, P.: Experiences with a novel fluidized bed system operating under vacuum conditions in Proceed. of Preworld Congress Particle Technology, Gifu, Japan, Sept. 17-18, 1990, 113-122.

18) Geldart, D.; Harby, N. and Wong, A.C.: Fluidization of cohesive powders in "The Role of Particle Interactions" in Powder Mechanics, preprint of Int. Symp. Eindhoven, Aug 29-31, 1983, p. 24 .

19) Luy, B.: Vakuum-Wirbelschicht, Grundlagen und Anwendungen in der Pharmazeutischen Technologie, PhD Thesis, The University of Basel, Switzerland, 1991.

20) Leuenberger, H.: The Impact of New Technologies: Vacuum-Fluidized Bed Granulation and Related Topics, Powrex Seminar, Proceedings of the Osaka Technical Conference, Osaka, Japan, July $22 / 23,1991$ p. 1 A-1 to $1 \mathrm{~A}-18$ (in Japanese).

\section{Author's short biography}

\section{Hans Leuenberger}

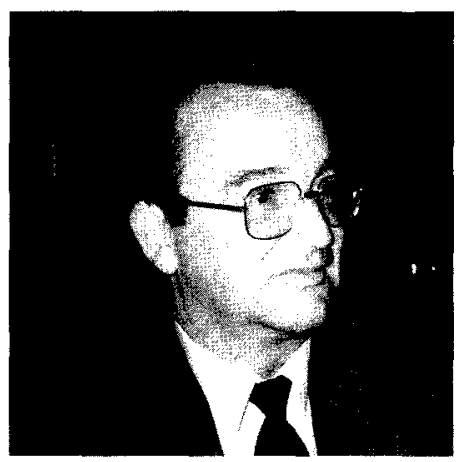

Since 1982 Prof. Hans Leuenberger is chairman of the Pharmaceutical Institute of the University of Basel and head of the Department of Pharmaceutical Technology and Biopharmacy. Prof. Leuenberger studied physics at the University of Basel, where he received his diploma in experimental physics in 1967 and his $\mathrm{PhD}$ in nuclear physics in 1971. From 1971 till 1982 he worked in the Pharmaceutical R+D labors at Sandoz Ltd., Basel. He served as head of a preformulation lab, research group leader and spent a stage at Sandoz (Spain) Ltd. as head of the Pharma ( $R+D)$ Department. With his nomination as an ordinary Professor at the University of Basel, he resigned in 1982 from his position at Sandoz Ltd. Prof. Leuenberger is actually president of the Scientific Council of the Swiss Academy of Engineering Sciences (SATW) and vicepresident of the Academy. He is a fellow of the American Association of Pharmaceutical Scientists (AAPS) and member of the expert group 12 (Pharm. Technology) of the European Pharmacopeia Commission.

Prof. Leuenberger and his coworkers are known for their contributions in the field of powder technology, controlled size enlargement of particles by power consumption measurement, compaction theory, novel process technologies such as vacuum-fluidized bed systems and spray-freeze drying at atmospheric pressure for pharmaceutical proteins, application of percolation theory and fractal geometry for the formulation of controlled release dosage forms as well as resorption models based on cell culture techniques in order to test novel drug delivery systems. 